\title{
NOVA SKED II: A behavioral notation language utilizing the Data General Corporation real-time disk operating system
}

\author{
STEVEN G. GILBERT \\ 49 Gilchrist A venue, Ottawa, Ontario KIY 0M9, Canada \\ and \\ DEBORAH C. RICE \\ Bureau of Chemical Safety, Food Directorate, Health Protection Branch, Health and Welfare Canada \\ Tunney's Pasture, Ottawa, Ontario KIA OL2, Canada
}

\begin{abstract}
A software system (NOVA SKED) was developed for the experimental control and collection of data from operant behavior experiments that is compatible with the Data General Corporation real-time disk operating system (RDOS). NOVA SKED is based on the SKED state notation language originally implemented on Digital Equipment Corporation PDP-8 series of minicomputers. The system includes a compiler written in FORTRAN, a multitasking run-time system that can be configured to run up to 30 stations, a data back-up system, standard data manipulation programs and subroutines, and user manuals.
\end{abstract}

The NOVA SKED ${ }^{1}$ software system, designed for the experimental control and collection of data from operant behavioral experiments, is fully compatible with the Data General Corporation real-time disk operating system (RDOS). This system integrates the powerful SKED state notation language with the flexible and well established RDOS operating system that supports a number of higher level languages and peripheral devices. RDOS, and hence, NOVA SKED, will run on a wide range of Data General computers, including the MICRONOVA with diskettes. This version of NOVA SKED offers several major revisions of the stand-alone NOVA SKED system previously reported (Gilbert \& Rice, 1978).

(1) The run-time system (RTS) is invoked directly from the disk, and compiled SKED programs are then loaded by the RTS as directed by the user. NOVA SKED can automatically return control to RDOS for data analysis when all sessions have finished. (2) A configuration procedure allows the user to run up to 30 stations and to choose between two types of $\mathrm{I} / \mathrm{O}$ hardware. (3) The I/O hardware bit assignments are independent of the RTS configuration, thus simplifying the accommodation of hardware changes. (4) Data collection is greatly improved in speed, flexibility, and syntax. (5) Keyboard commands have been expanded to take advantage of the operating system. (6) Supporting software includes data manipulation programs and several FORTRAN subroutines. (7) All documentation is stored in disk files for easy reproduction.

\section{THE COMPILER}

The SKED compiler, a two-pass compiler written in FORTRAN IV, generates binary files for input to the

The authors would like to thank Geoffrey Inglis for several discussions on the logic of the SKED language and run-time system.
RTS. On the first pass, the compiler does extensive syntax checking, generating error messages as errors are found. The second pass completes the NOVA SKED binary, and an optional third pass generates a commented listing of the SKED binary. The NOVA SKED compiler requires $18 \mathrm{~K}$ words. Since RDOS requires approximately $14 \mathrm{~K}$ words, a minimum system to run NOVA SKED is $32 \mathrm{~K}$ words.

\section{THE RUN-TIME SYSTEM}

The NOVA SKED RTS is fully compatible with RDOS and mapped RDOS (MRDOS), taking advantage of the flexible file structure and system commands. The SKED software system is used to its maximum under MRDOS. Under such a system, the SKED RTS can be run in the foreground with highest priority, while program development, FORTRAN or SKED compilations, or data analysis can proceed in the background. The RTS will run in as little as $7 \mathrm{~K}$ words of memory but can automatically adjust to the assigned memory up to a maximum of $31 \mathrm{~K}$ words. A large amount of assigned memory and the use of reentrant SKED binaries makes it possible to operate up to 30 stations.

There are two points to consider when running a large number of stations. (1) Any number of stations may be run by one program, but the maximum number of SKED programs in memory at one time is 16. (2) Each station that uses a disk data file requires 512 words of memory for two data buffers. An option is being considered that uses only one data buffer ( 256 words) but requires the data to be sorted after the session ends.

The RTS size and capabilities can be configured to an experimental environment by a SKED generation procedure. The following are configured in the RTS: (1) the number of stations (1-30), (2) hardware or 
software multiply/divide, (3) the type of $1 / 0$ interface (chassis subsystem or digital I/O boards), and (4) the alternative that unwanted special functions may be excluded.

Each time the RTS is started, an I/O table is read and saved in memory. The $\mathrm{I} / \mathrm{O}$ table is a disk file, generated by a question-and-answer support program, that contains the device codes (DIO boards) or card numbers (chassis subsystem), the number of stations, the number of input and output bits per station (up to 256 of each), and the physical bit assignments. Since the $I / O$ configuration is independent of the RTS, any number of $I / O$ files can be created. The RTS assumes a default $1 / 0$ file, but a switch allows any file name to be specified when the RTS is invoked. The I/O table disk file can be modified by the support program to reflect bit changes, and the number of stations may be expanded. After being read by the RTS, the I/O table can be inspected or modified from the keyboard.

The RTS supports an interactive batch system that allows the user to submit a disk file of SKED commands from the keyboard. Batch is typically used to load the programs and header information, and then to start the stations for a set of subjects run on a routine basis. This could include either running a group of subjects in separate stations or running the subjects sequentially in one station. In the latter case, batch would be halted or suspended by an internal command after each subject and then restarted by the user for the next session. In addition, the batch file may contain nonexecuting comment lines and messages to be printed to the console.

In the SKED language, F3 functions give the RTS additional flexibility by providing a means of expansion. F3s are executed as part of an output expression and perform operations such as data output to peripheral devices, variable manipulations, and operations that are cumbersome to program in state notation. The system is designed so that F3s may be added with no changes to the compiler and with only a one-word addition to an RTS table. Keyboard commands can be added in the same manner. New F3s have improved data collection (see below) and allow messages and variables to be typed from SKED programs.

\section{DATA COLLECTION}

The collection and analysis of data are much easier in the current version of disk-based SKED. The RDOS file structure allows each station using disk "write" functions to have a separate data file that contains a nine-word header followed by a continuous list of data written in binary. To insure that no data are lost while data are being written to the disk, each station has two 256-word data buffers that are created when a station is loaded into memory. When a station ends, the last buffer is written, the data file is closed, and the data buffers are removed from memory. Disk files are opened automatically with a name created from the RDOS system date and the station header according to the following format: DEXMMDDYY.SS, where $D$ is a single letter that may be entered with the experiment number (D is the default letter); EX is the experiment number; MMDDYY is the date in month, day, year; and SS is the subject number extension. This name format was chosen for several reasons: (1) Most RDOS commands allow the date to be represented symbolically with \%DATE\%. (2) The variable first letter allows an additional level of grouping. This letter could be used to identify individual users in a multiuser environment or to separate different species of animals. (3) Grouping by experiment number or subject number is simplified.

Data collection by coding interevent times (IETs) has been made more efficient. The improvements include a simplified syntax and a faster way of handling IET collection that makes the operation of a larger number of stations possible. Other methods of data collection, such as using event counters, are supported and can now be combined with IET collection. A new F3 function allows variables and constants to be coded and written to the data file in the same format used in IET collection. Regardless of the type of data collection, data files are written and closed automatically.

The NOVA SKED system also includes a data backup and retrieval system for those with access to magnetic tape. Data is stored in the RDOS DUMP format, which allows a mag-tape file to contain any number of disk files. Individual files or groups of files are readily returned to the disk with the RDOS LOAD command.

Although data analysis is often unique to a particular experiment, a number of general-purpose FORTRAN subroutines and support programs are available. Several programs have been designed to convert the binary data into a readable format, including programs that convert binary data files to ASCII and then back to binary, print the IETs and codes, and total and label all IET codes. The FORTRAN subroutines include routines to format and print a data file header, return the IET and code in separate words, read the RDOS command line, and create file names and line-printer plotting routines.

\section{DOCUMENTATION}

The NOVA SKED software has been carefully documented so that retrieval and maintenance can be accomplished quickly and easily. All documentation has been typed into disk files that can be listed by a special formatting and indexing program. These documents include The NOVA SKED Users Manual, which describes the language and systems in detail; NOVA SKED Overview, a reference manual giving an overview of the SKED software organization; NOVA SKED Systems Manual; and manuals that describe the 
support programs and subroutines with detailed calling instructions. The NOVA SKED RTS, compiler, support programs, and documentation are available on mag tape from Steven Gilbert.

\section{NOTE}

1. NOVA is a registered trademark of Data General Corporation, Southboro, Massachusetts. SKED is a registered trademark of State System, Inc. for its brand of computer interface, software, and service associated therewith.

\section{REFERENCES}

GILBERT, S. G., \& RICE, D. C. NOVA SKED: A behavioral notation language for Data General minicomputers. Behavior Research Methods \& Instrumentation, 1978, 10, 705-709.

(Received for publication July 18, 1978; revision accepted August 15, 1978.) 